\title{
Isovitexin, a Potential Candidate Inhibitor of Sortase A of Staphylococcus aureus USA300
}

\author{
Dan $\mathrm{Mu}^{1 \dagger}$, Hua Xiang ${ }^{2 \dagger}$, Haisi Dong ${ }^{1}$, Dacheng Wang ${ }^{1 *}$, and Tiedong Wang ${ }^{1 *}$ \\ ${ }^{1}$ College of Animal Science, Jilin University, Changchun, P.R. China \\ ${ }^{2}$ College of Animal Science and Technology, Jilin Agricultural University, Changchun 130118, P.R. China
}

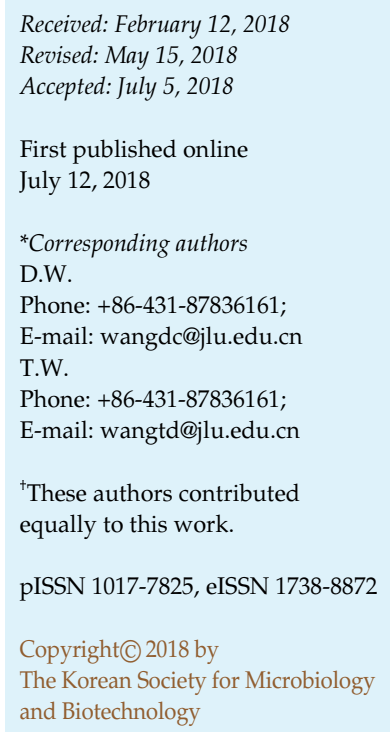

Staphylococcus aureus causes a broad variety of diseases. The spread of multidrug-resistant $S$. aureus highlights the need to develop new ways to combat $S$. aureus infections. Sortase A (SrtA) can anchor proteins containing LPXTG binding motifs to the bacteria surface and plays a key role in $S$. aureus infections, making it a promising antivirulence target. In the present study, we used a SrtA activity inhibition assay to discover that isovitexin, a Chinese herbal product, can inhibit SrtA activity with an $\mathrm{IC}_{50}$ of $28.98 \mu \mathrm{g} / \mathrm{ml}$. Using a fibrinogen-binding assay and a biofilm formation assay, we indirectly proved the SrtA inhibitory activity of isovitexin. Additionally, isovitexin treatment decreased the amount of staphylococcal protein A (SpA) on the surface of the cells. These data suggest that isovitexin has the potential to be an anti-infective drug against $S$. aureus via the inhibition of sortase activity.

Keywords: Staphylococcus aureus, antivirulence, isovitexin, inhibitor, sortase A

\section{Introduction}

Staphylococcus aureus is a major human commensal and opportunistic pathogen and causes a broad spectrum of human diseases, ranging from moderate soft tissue infections to more severe infections such as endocarditis, pneumonia, septic shock, necrotizing fasciitis, osteomyelitis and blood infections [1-3]. This pathogen is also one of the leading reasons of both hospital- and community-acquired infectious diseases [4]. The importance of $S$. aureus has been amplified owing to the rise and spread of multidrug-resistant (MDR) S. aureus, such as vancomycin-resistant $S$. aureus (VRSA) and methicillin-resistant S. aureus (MRSA), which cause increased morbidity and mortality and present a clinical challenge [5].

Traditional approaches for treating bacterial infection rely primarily on the application of antibiotics, which disrupt the growth cycle by inhibiting the synthesis or activity of essential proteins or by preventing the assembly of key constituents such as cell wall [6]. Although antibiotics can eliminate most of the target bacteria, they also exert remarkable selective pressure on bacterial evolutionary adaptation, resulting in the development of bacterial resistance [7]. Importantly, broad-spectrum antibiotics also induce disordered host intestinal microbiota and result in diarrhoea and intestinal infection of the host [8]. Hence, alternative therapies involving antivirulence agents have aroused great attention. S. aureus uses two types of virulence factors, adhesins and toxins, to establish infection or cause pathological changes in the human host [9]. Adhesins are covalently anchored cell surface proteins that are primarily responsible for mediating infection processes such as attachment, colonization, cell-cell interactions, the invasion of host endothelial tissues, the evasion of host immunity and iron acquisition [10]. Interference with virulenceassociated surface protein anchoring has emerged as a promising strategy to combat $S$. aureus diseases since those virulence factors are not essential for the survival of bacteria [11, 12]. The cell surface proteins containing sorting LPXTG motifs are secreted as precursors and are 
covalently anchored to the peptidoglycan layer of the gram-positive cell wall by means of a universal sorting mechanism mediated by thesortase transpeptidase [13]. Sortase A (SrtA) plays a pivotal role in pathogenesis of S. aureus by modulating the sorting of the virulenceassociated protein to the cell surface [14]. It is an appealing target for drug development for $S$. aureus infections.

Natural products have been considered a major source of pharmaceutical leads and therapeutic drugs. They have functional diversity and are being exploited for a variety of novel bactericidal or antivirulence agents against bacterial infections [15]. Among them, naturally existing flavonoids are worthy of special attention because they possess not only a wide range of pharmacological effects but also the potential to inhibit microbial virulence [16, 17]. Isovitexin, apigenin-6-C-D-glucopyranoside, is a naturally derived flavonoid. It has been proven to possess various pharmacological effects such as antioxidant, antineoplastic, anticancer, anti-inflammatory, antihyperalgesic, cardiovascular system protection and neuroprotection effects [18-21]. In the present study, we found that isovitexin could inhibit S. aureus SrtA activity without affecting bacterial growth and survival, indicating that isovitexin could be employed as a prospective lead compound for further development of drugs against $S$. aureus.

\section{Materials and Methods}

\section{Bacteria, Chemicals and Growth Conditions}

S. aureus USA300 strain BAA-1717 (American Type Culture Collection) as well as its $s r t A$ mutant strain $(\triangle \operatorname{srt} A)$ was used throughout this study. The $\Delta s r t A$ strain was constructed by a method described previously [22]. Cells of $S$. aureus were subcultured in brain heart infusion (BHI) broth at $37^{\circ} \mathrm{C}$. Isovitexin was purchased from ChemBest Research Laboratories Ltd (China). All other compounds used were obtained from SigmaAldrich (China). The peptide substrate Abz-LPATG-Dap(Dnp)$\mathrm{NH}_{2}$ (Abz: ortho-aminobenzoic acid; Dnp: 2,4-dinitrophenyl) was synthesized by GL Biochem (China).

\section{Cloning, Overexpression, and Purification of SrtA}

The genomic DNA of $S$. aureus USA300 was isolated and used as the template DNA in PCR reactions. The srtA gene lacking the transmembrane domain (N1 - 59) was amplifiedby PCR using the primers 5'-GGGAATTCCATATGCAAGCTAAACCTCAAATTC CG-3' (forward) and 5'-CGCGGATCCTTATTTGACTTCTGTAGC TACAAAGA-3' (reverse). The resulting amplified fragment was digested with BamHI and XhoI and ligated into plasmid pET28adigested with the same restriction enzyme. The recombinant plasmid was transformed into the Escherichia coli strain BL21 (Novagen) for the overexpression of the SrtA. Production of recombinant SrtA protein was induced at mid-log phase with $1 \mathrm{mM}$ isopropylthio- $\beta$-D-galactoside (IPTG) for $4 \mathrm{~h}$ at $16^{\circ} \mathrm{C}$. Recombinant His-tagged $\mathrm{SrtA}_{\triangle \mathrm{N} 59}$ was purified by the $6 \times \mathrm{His} / \mathrm{Ni}-$ NTA system as described previously [23].

\section{Analysis of Anti-S. aureus Activity of Isovitexin}

The minimum inhibitory concentration (MIC) for the isovitexin was determined through broth dilution methods as suggested by Kim ES [24]. In brief, overnight bacterial cultures were diluted to an $\mathrm{OD}_{600}$ (optical density at $600 \mathrm{~nm}$ ) of 0.3 with BHI broth containing different concentrations of isovitexin $(0,16,32,64,128$, 256,512 , and $1,024 \mu \mathrm{g} / \mathrm{ml}$ ) and were cultured for $16 \mathrm{~h}$ at $37^{\circ} \mathrm{C}$. Absorbance values $\left(\mathrm{OD}_{600}\right)$ were measured using a multimode microplate reader (Infinite F500, Tecan, China). For growth curve plotting, S. aureus from overnight cultures was diluted back 1:100 in sterile BHI broth with or without $256 \mu \mathrm{g} / \mathrm{ml}$ isovitexin and were grown at $37^{\circ} \mathrm{C}$ with shaking for $24 \mathrm{~h}$. Then absorbance at $600 \mathrm{~nm}$ was measured at different time intervals.

\section{SrtA Activity Assay}

Sortase activity was determined by the fluorescence resonance energy transfer (FRET) method according to a published protocol described previously $[25,26]$. The synthetic peptide Abz-LPATGDap(Dnp)- $\mathrm{NH}_{2}$ was used as the fluorescent internally quenched substrate. The assay was conducted in the wells of a 96-well black plate (PerkinElmer). Wells contained $10 \mu \mathrm{M}$ purified SrtA and different concentrations of isovitexin in a final volume of $300 \mu \mathrm{l}$ of the reaction buffer $(50 \mathrm{mM}$ Tris $\cdot \mathrm{HCl}, 150 \mathrm{mM} \mathrm{NaCl}$ and $5 \mathrm{mM}$ $\mathrm{CaCl}_{2}$, pH 7.5). Wells contained all the above, except for the test sample, which was used as a blank control. The reaction was carried out for $30 \mathrm{~min}$ at $37^{\circ} \mathrm{C}$. Then $50 \mu \mathrm{M}$ substrate peptide was added and incubated for a further hour, and fluorescence was read at $495 \mathrm{~nm}$ emission wavelengths with an excitation wavelength of $350 \mathrm{~nm}$. Inhibitory rates of isovitexin on SrtA activities were determined by the formula: $100 \% \times(C-T) / C$, where $C$ is the fluorescent value of the untreated group, and $\mathrm{T}$ is the fluorescent value of the experimental group.

\section{Adherence of $S$. aureus to Immobilized Fibrinogen}

$S$. aureus wild-type (WT) and $\Delta s r t A$ strains were grown overnight, back-diluted 1:1000 in sterile BHI medium and cultured to an $\mathrm{OD}_{600}$ of 0.5 in the presence of isovitexin or DMSO with shaking at $37^{\circ} \mathrm{C}$. The $\triangle s r t A$ strain grown in BHI medium supplemented with $256 \mu \mathrm{g} / \mathrm{ml}$ isovitexin served as the positive control. The untreated group served as the negative control. The cultures were centrifuged at 3,000 $\mathrm{g}$ for $5 \mathrm{~min}$, washed three times with phosphate-buffered saline (PBS) and diluted to an $\mathrm{OD}_{600}$ of 1.0. Wells of Costar flat-bottomed microtiter plate were coated with $20 \mu \mathrm{g} / \mathrm{ml}$ bovine fibrinogen $(\mathrm{Fg})$ overnight at $4^{\circ} \mathrm{C}$. The plates were rinsed two times and blocked for $2 \mathrm{~h}$ with $5 \%$ bovine serum albumin (BSA). The plates were washed three times with PBS, and $100 \mu \mathrm{l}$ of the cell suspensions was added to the fibrinogen-coated plates. Following incubation for $2 \mathrm{~h}$ at $37^{\circ} \mathrm{C}$, the suspension 
was discarded, and the wells were washed three times with PBS. Then the bound bacteria were fixed with $2 \%(\mathrm{v} / \mathrm{v})$ glutaraldehyde for $30 \mathrm{~min}$. After washing once, the bacteria were stained with $12.5 \mathrm{~g} / \mathrm{l}$ crystal violet for $15 \mathrm{~min}$. Then the plates were washed again with PBS and allowed to dry overnight. The bound dye was eluted with $95 \%$ ethanol and the amount of dye was then determined using a microplate reader. The percent inhibition of isovitexin on the adhesion of $S$. aureus is determined by the formula: $100 \% \times(\mathrm{C}-\mathrm{T}) / \mathrm{C}$, where $\mathrm{C}$ is the absorbance value of the non-treatment group, and $\mathrm{T}$ is the absorbance value of the drug treatment group.

\section{Crystal Violet Biofilm Assay}

A static biofilm assay of $S$. aureus was carried out in flatbottomed, microtiter plates (BD, Falcon) based on a method described previously [27]. Briefly, bacteria cultures were suspended into $200 \mu \mathrm{l}$ of BHI medium supplemented with $3 \%(\mathrm{w} / \mathrm{v})$ sucrose to an absorbance of 0.5 at $600 \mathrm{~nm}$ and grown statically with or without isovitexin at $37^{\circ} \mathrm{C}$ for $24 \mathrm{~h}$. The medium was then discarded, and the plates were washed thrice with PBS to remove residual planktonic cells. To quantify the biofilm formation, the adherent cells were stained with $0.1 \%$ crystal violet for $20 \mathrm{~min}$. The wells were thoroughly rinsed with sterile deionized water to remove unbound dye. The bound dye was eluted in $300 \mu \mathrm{l}$ of $33 \%$ acetic acid. The absorbances at $595 \mathrm{~nm}$ were determined using a microplate reader. The $\Delta s r t A$ strain grown in BHI medium served as a positive control. The untreated group served as a negative control.

\section{Staphylococcal Protein A (SpA) Display Analysis}

S. aureus WT and $\Delta s r t A$ strains were grown overnight and then back-diluted 1:1000 in fresh $\mathrm{BHI}$ medium and cultured to $\mathrm{OD}_{600}$ between $0.8-1.0$ with isovitexin or DMSO at $37^{\circ} \mathrm{C}$. The untreated $\triangle$ srtA strain served as a positive control for SpA inhibition. The WT group served as a negative control. The cells from each culture were collected by centrifugation at 3,000 $\mathrm{g}$ for $5 \mathrm{~min}$, and the bacteria were washed twice with PBS and fixed in a $4 \%$ formaldehyde solution for $20 \mathrm{~min}$. After washing twice in PBS, the bacteria were stained with a 1:100 dilution of polyclonal FITCconjugated goat anti-rabbit immunoglobulin G (IgG) (eBioscience) for $2.5 \mathrm{~h}$ at $25^{\circ} \mathrm{C}$. At last the bacteria were washed twice and loaded on poly-L-lysine-coated glass slides. The images were obtained by a confocal microscope (Olympus, China).

\section{Results}

\section{Isovitexin Inhibits the Activity of SrtA}

To determine if isovitexin had an inhibitory effect on SrtA activity, a FRET assay was performed with the selfquenched fluorescent peptide Abz-Leu-Pro-Glu-Thr-GlyLys-Dap(Dnp)- $\mathrm{NH}_{2}$, which contains the LPXTG-motif of the SrtA substrate protein. After cleavage by SrtA, the fluorophore $\mathrm{Abz}$ within the peptide is separated from the quencher Dnp, which causes an increase in fluorescence. We incubated purified $\mathrm{Srt}_{\Delta \mathrm{N} 59}$ with the fluorescent peptide substrate at various concentrations of isovitexin in the reaction buffer, and the results showed that the addition of isovitexin decreased the fluorescence signal in a dosedependent manner, indicating that isovitexin possesses potent SrtA inhibitory activity. The half-maximal inhibitory concentration $\left(\mathrm{IC}_{50}\right)$ value of isovitexin was $28.98 \mu \mathrm{g} / \mathrm{ml}$ (Fig. 1A).
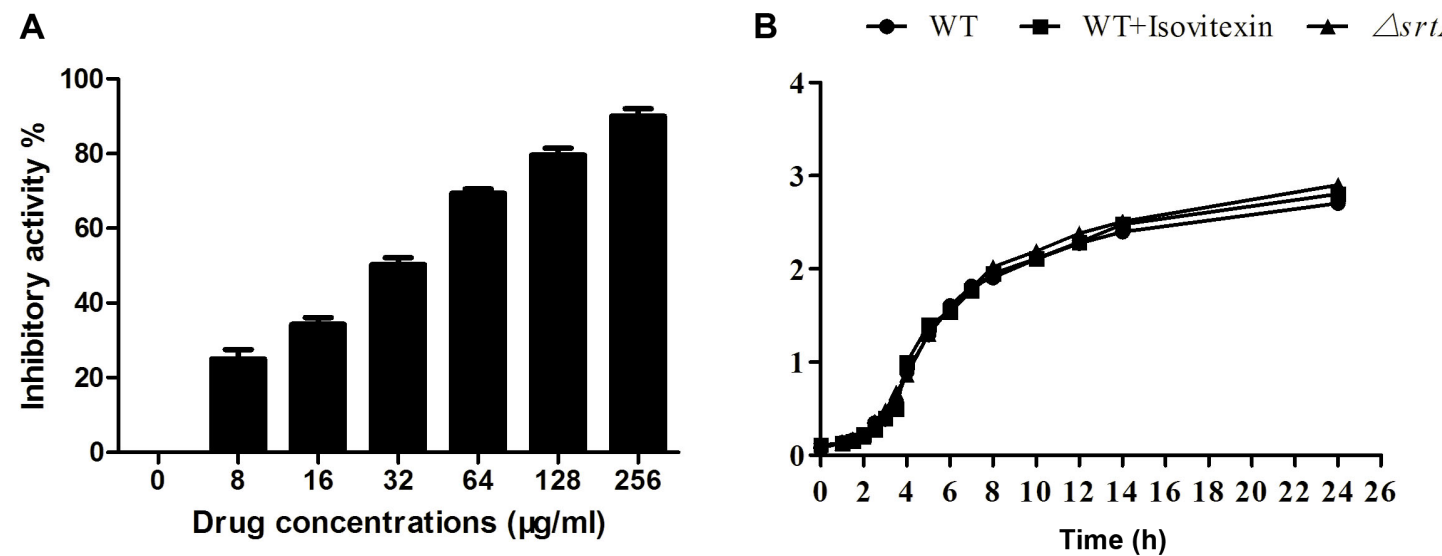

Fig. 1. Inhibitory effect of isovitexin against SrtA in vitro.

(A) The reaction solutions contained SrtA, the synthetic fluorescent peptide substrate Abz-LPATG-Dap(Dnp)-NH $\mathrm{N}_{2}$ and various concentrations of isovitexin. (B) Effect of isovitexin on the growth of S. aureus. The WT strain was cultured in BHI broth with $256 \mu \mathrm{g} / \mathrm{ml}$ isovitexin (square) or without isovitexin (circle). The $\Delta s r t A$ strain served as a positive control (triangle). The graphs indicate the averages of three independent experiments. 


\section{Isovitexin Has No Influence on S. aureus Growth}

To test whether isovitexin can inhibit the growth of S. aureus, the MIC of isovitexin against S. aureus was measured. The MIC value of isovitexin against S. aureus WT strain was $>1,024 \mu \mathrm{g} / \mathrm{ml}$. The growth curves of the $S$. aureus showed that the growth rates of the $S$. aureus WT strain treated with isovitexin and the $S$. aureus $\Delta s r t A$ strain were similar to that of the $S$. aureus WT strain (Fig. 1B). These results suggest that isovitexin doesn't affect $S$. aureus proliferation.

\section{Isovitexin Reduces $S$. aureus Adhesion to Fibrinogen}

An earlier study has shown that the inhibitors of SrtA can inhibit $S$. aureus cell adhesion to fibrinogen (Fg)- or fibronectin-coated plates [28]. Therefore, we used the $S$. aureus adhesion assay to test whether isovitexin inhibits the activity of the SrtA enzyme. As expected, the result showed that the $S$. aureus adhesion to Fg-coated plates was inhibited by isovitexin in a dose-dependent manner (Fig. 2). The $S$. aureus $\triangle$ srtA strain exhibited a minimum binding capacity to Fg-coated surfaces of $9.3 \pm 1.7 \%$, and the fibrinogen binding capacity of the $S$. aureus WT strain treated with $32,64,128$, or $256 \mu \mathrm{g} / \mathrm{ml}$ of isovitexin was measured, and the adhesion rates were $86.3 \pm 5.8 \%, 54.3 \pm$ $4.0 \%, 34.7 \pm 4.0 \%$, and $25.3 \pm 4.6 \%$, respectively.

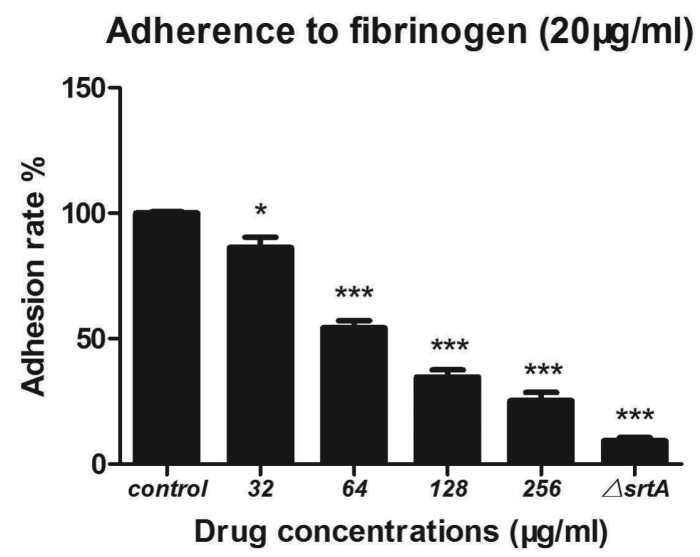

Fig. 2. Inhibition of $S$. aureus binding to immobilized Fg by isovitexin.

The S. aureus WT strain was cultured in BHI medium supplemented with DMSO or various concentrations of isovitexin. The S. aureus $\Delta s r t A$ strain treated with $256 \mu \mathrm{g} / \mathrm{ml}$ isovitexin served as a positive control. The control group was the non-treatment group. The bacteria were added into Fg-coated microtiter wells and incubated for $2 \mathrm{~h}$ at $37^{\circ} \mathrm{C}$. The attached bacteria in microtiter plates were stained with crystal violet. Data are the means \pm SD of three replicates. The symbol "** represents $p<0.05$, and the symbol "****" represents $p<0.0001$.

\section{Isovitexin Reduces Biofilm Formation}

$S$. aureus is capable of forming biofilms on many tissues or implanted medical devices. S. aureus biofilms often cause chronic infections since that bacteria in biofilms can evade antibiotic eradication and host immune responses [29]. A previous study revealed that the SrtA knockout strain showed significant reduction of biofilm formation of MRSA strains [30]. As expected, the results of biofilm formation assay showed $S$. aureus biofilm formation was inhibited by isovitexin. Further quantitative analysis showed that $256 \mu \mathrm{g} / \mathrm{ml}$ isovitexin inhibited biofilm formation by $\geq 61 \%$ (Fig. 3).

\section{Isovitexin Reduces SpA Display on the Surface of S. aureus}

SpA, a major virulence factor, is expressed by almost all clinical isolates of $S$. aureus [31]. It is secreted by the general secretory pathway and is displayed on the surface of bacteria by SrtA during secretion [32, 33]. In this assay, $S$. aureus cultured with or without isovitexin was stained with goat anti-rabbit IgG labelled with FITC to detect SpA on the surface of the bacteria. The result showed that the S. aureus WT strain cultured in the presence of $256 \mu \mathrm{g} / \mathrm{ml}$ isovitexin revealed a very faint distribution of $\mathrm{SpA}$, in

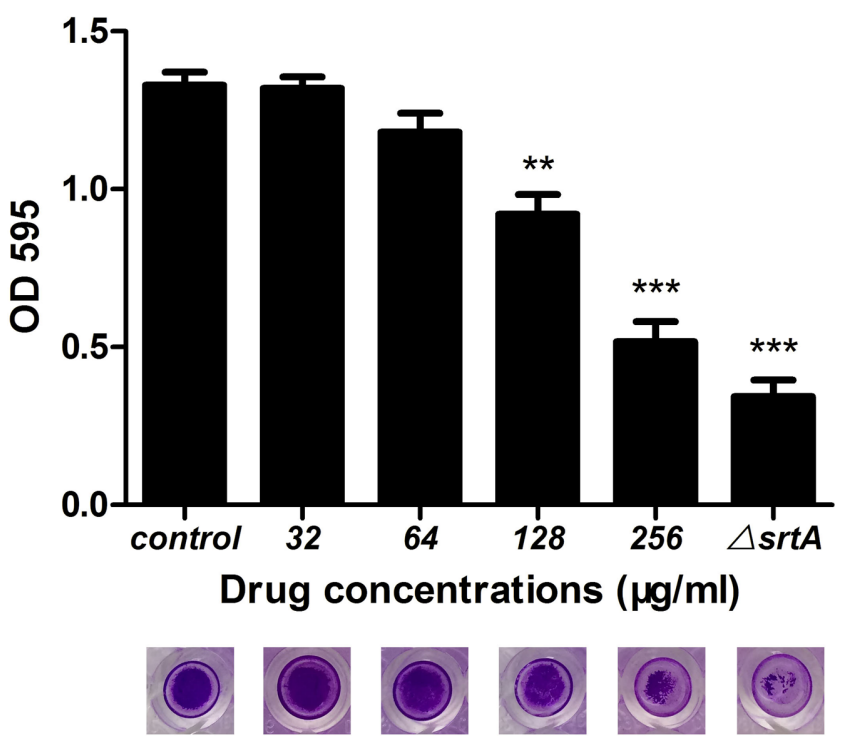

Fig. 3. Inhibition of the biofilm formation by isovitexin.

The $S$. aureus WT strain was incubated in BHI broth supplemented with various concentrations of isovitexin for $24 \mathrm{~h}$. The biofilm grown in microtiter plates was stained with crystal violet. The data were obtained from three replicates and were presented as the means \pm SD. The symbol "**" represents $p<0.05$, and the symbol "***" represents $p<0.0001$. 


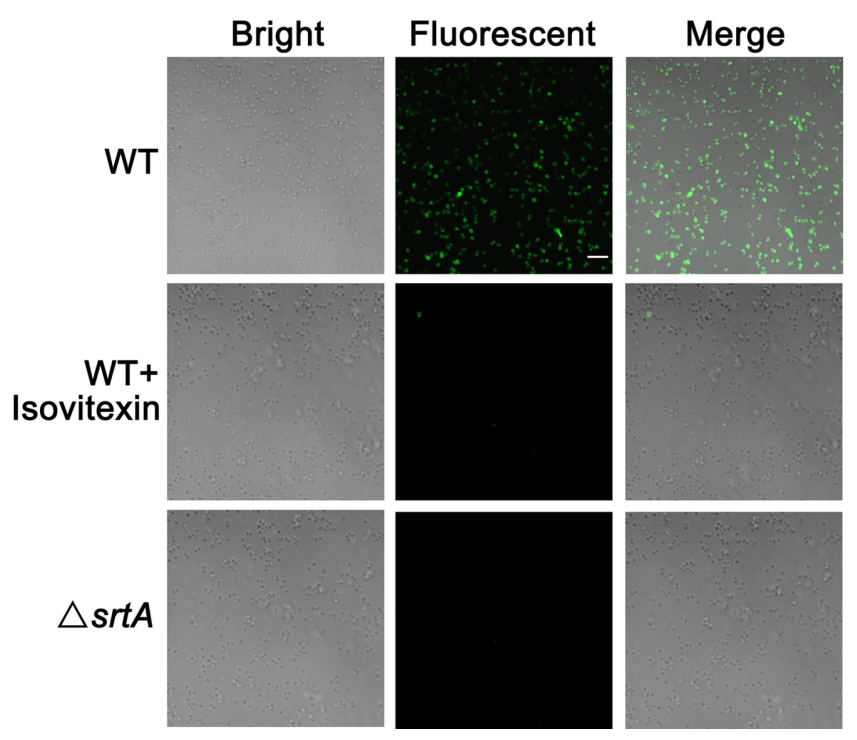

Fig. 4. Decrease of SpA display on the $S$. aureus surface by isovitexin.

The S. aureus WT strain treated with $256 \mu \mathrm{g} / \mathrm{ml}$ isovitexin was cultured in BHI broth. The untreated S. aureus WT strain and the $S$. aureus $\Delta$ srtA strain were used as a negative control and a positive control for SpA inhibition respectively. The cells were stained with FITC-labeled IgG. Fluorescence images were collected by a confocal laser-scanning microscopy. Scale bar indicates $5 \mu \mathrm{m}$.

comparison with the distributions visible on the S. aureus $\Delta s r t A$ strain and the untreated S. aureus WT strain (Fig. 4). These consequences indicate that isovitexin can disturb the display of SpA on the bacterial surface by inhibiting SrtA.

\section{Discussion}

The emergence and spread of $S$. aureus strains such as MRSA, VRSA, and multiple-drug-resistant $S$. aureus (MDRSA) pose a considerable challenge to the treatment of clinical S. aureus infections [34]. The conventional antibiotics have targets associated with bacterial growth and survival, which exert substantial selective pressure on the adaptive evolution of the bacteria and promote the development of antibiotic resistance [35]. Therefore, it is imperative to develop alternative strategies to combat $S$. aureus infections.

$S$. aureus can anchor at least 25 LPXTG proteins on its surface by SrtA, such as coagulation factorA and B (ClfA and $\mathrm{ClfB}$ ), fibronectin-binding protein $\mathrm{A}$ and $\mathrm{B}$ (FnbpA and FnbpB), and SpA [36]. These cell wall-anchored (CWA) surface proteins are essential virulence factors and possess various functions, including the adhesion to host tissues, the invasion of non-phagocytic cells, the evasion of immune responses, nutrient acquisition, and biofilm formation [37]. SrtA, an intensively studied virulence factor, can anchor CWA proteins to its surface and plays a vital role in S. aureus infections. Research showed that $s r t A$ gene knockout or inhibition of SrtA in $S$. aureus by inhibitors resulted in the defective display of LPXTG proteins and severe virulence defects in the establishment of infection [38]. Therefore, screening SrtA inhibitors is an especially appealing strategy for treating $S$. aureus infections. Chemical extracts from natural products are of great variety and have chemical diversity. They are a major source of therapeutic agents in some bacteria infectious diseases. Early studies have shown that flavonoids are a group of promising natural compounds against SrtA [39, 40]. Recently, our research work has primarily focused on discovering potent flavonoid compounds from Chinese herbal products against important virulence factors in S. aureus $[41,42]$. As a continuation of that work, in this study, we found that isovitexin, a natural flavonoid active ingredient without growth inhibitory activity against S. aureus, can significantly inhibit the activity of SrtA. The Fg-binding assay indicates that isovitexin can interfere with SrtA activity and can affect the adhesion of S. aureus cells to Fg-coated surface (Fig. 2). LPXTG-containing surface proteins such as biofilm-associated protein (Bap) and/S. aureus surface protein (SasG), which are identified mediators of $S$. aureus biofilm development $[43,44]$. Further research showed that the $s r t A$ gene mutation had an impact on biofilm accumulation by clinical MRSA strains [30]. The results of our biofilm formation assay show that isovitexin can inhibit the biofilm formation of S.aureus USA300, indirectly reflecting the inhibition of sortase activity (Fig. 3). $\mathrm{SpA}$, as a kind of multifunctional surface protein of S. aureus, binds to the Fc portion of IgG and to the von Willebrand factor (vWF) during infection [45]. It is synthesized in the cytoplasm and then translocated onto the staphylococcal surface by SrtA via transpeptidation and transglycosylation reactions [46]. An earlier study has shown that $S$. aureus $\operatorname{srt} A$ mutants are incapable of anchoring SpA to cell surfaces [47]. As expected, the SpArelated fluorescence analysis showed that isovitexin reduced the amount of SpA on the bacterial surface by inhibiting SrtA activity (Fig. 4).

In summary, our results show that isovitexin has potent inhibitory activity against SrtA in vitro and has no anti$S$. aureus growth activity. It will be meaningful to develop antivirulence drugs based on isovitexin. 


\section{Acknowledgments}

This work was supported by The National Key Technology R \& D Program, China (2016YFD05013).

\section{Conflict of Interest}

The authors have no financial conflicts of interest to declare.

\section{References}

1. Ericson JE, Popoola VO, Smith PB, Benjamin DK, Fowler VG, Jr BD, et al. 2015. Burden of invasive Staphylococcus aureus infections in hospitalized infants. JAMA Pediatr. 169: 11981205 .

2. Defres S, Marwick C, Nathwani D. 2009. MRSA as a cause of lung infection including airway infection, communityacquired pneumonia and hospital-acquired pneumonia. Eur. Respir. J. 34: 1470-1476.

3. Ippolito G, Leone S, Lauria FN, Nicastri E, Wenzel RP. 2010. Methicillin-resistant Staphylococcus aureus: the superbug. Int. J. Infect. Dis. 14: S7-11.

4. Boyle-Vavra S, Daum RS. 2007. Community-acquired methicillin-resistant Staphylococcus aureus: the role of PantonValentine leukocidin. Lab. Invest. 87: 3-9.

5. Zetola N, Francis JS, Nuermberger EL, Bishai WR. 2005. Community-acquired meticillin-resistant Staphylococcus aureus: an emerging threat. Lancet Infect Dis. 5: 275-286.

6. Woodin KA, Morrison SH. 1994. Antibiotics: mechanisms of action. Pediatr. Rev. 15: 440-447.

7. Rasko DA, Sperandio V. 2010. Anti-virulence strategies to combat bacteria-mediated disease. Nat. Rev. Drug Discov. 9: 117-128.

8. Sekirov I, Tam NM, Jogova M, Robertson ML, Li Y, Lupp C, et al. 2008. Antibiotic-induced perturbations of the intestinal microbiota alter host susceptibility to enteric infection. Infect. Immun. 76: 4726-4736.

9. Smeltzer MS. 2016. Staphylococcus aureus pathogenesis: the importance of reduced cytotoxicity. Trends Microbiol. 24: 681-682.

10. Foster TJ, Höök M. 1998. Surface protein adhesins of Staphylococcus aureus. Trends Microbiol. 6: 484-488.

11. Cegelski L, Marshall GR, Eldridge GR, Hultgren SJ. 2008. The biology and future prospects of antivirulence therapies. Nat. Rev. Microbiol. Nature Rev. Microbiol. 6: 17-27.

12. Clatworthy AE, Pierson E, Hung DT. 2011. Targeting virulence: a new paradigm for antimicrobial therapy. Nat. Chem. Biol. 3: 541-548.

13. Kruger RG, Otvos B, Frankel BA, Bentley M, Patrick Dostal A, Mccafferty DG. 2004. Analysis of the substrate specificity of the Staphylococcus aureus sortase transpeptidase SrtA.
Biochemistry 43: 1541-1551.

14. Maresso AW, Schneewind O. 2008. Sortase as a target of anti-infective therapy. Pharmacol. Rev. 60: 128-141.

15. Silva LN, Zimmer KR, Macedo AJ, Trentin DS. 2016. Plant natural products targeting bacterial virulence factors. Chem. Rev. 116: 9162-9236.

16. Kong C, Neoh HM, Nathan S. 2016. Targeting Staphylococcus aureus toxins: a potential form of anti-virulence therapy. Toxins (Basel) 8: 72.

17. Payne DJ, Gwynn MN, Holmes DJ, Pompliano DL. 2007. Drugs for bad bugs: confronting the challenges of antibacterial discovery. Nat. Rev. Drug Discov. 6: 29-40.

18. He M, Min JW, Kong WL, He XH, Li JX, Peng BW. 2016. A review on the pharmacological effects of vitexin and isovitexin. Fitoterapia 115: 74-85.

19. Ganesan K, Xu B. 2017. Molecular targets of vitexin and isovitexin in cancer therapy: a critical review. Ann. NY Acad. Sci. 1401: 102-113.

20. GA D, AR S, LS B, RC B, PP M, BS V, et al. 2015. Redoxactive profile characterization of remirea maritima extracts and its cytotoxic effect in mouse fibroblasts (L929) and melanoma (B16F10) cells. Molecules 20: 11699-11718.

21. Lee CY, Chien YS, Chiu TH, Huang WW, Lu CC, Chiang JH, et al. 2012. Apoptosis triggered by vitexin in U937 human leukemia cells via a mitochondrial signaling pathway. Oncol. Rep. 28: 1883-1888.

22. Chen F, Liu B, Wang D, Wang L, Deng X, Bi C, et al. 2014. Role of sortase A in the pathogenesis of Staphylococcus aureus-induced mastitis in mice. FEMS Microbiol. Lett. 351: 95-103.

23. Changsheng $\mathrm{Lu}$, Zhu J, Wang $\mathrm{Y}$, Umeda A, Cowmeadow RB, Lai E, et al. 2007. Staphylococcus aureus sortase A exists as a dimeric protein in vitro. Biochemistry 46: 9346-9354.

24. Kim ES, Kang SY, Kim YH, Lee YE, Choi NY, You YO, et al. 2015. Chamaecyparis obtusa essential oil inhibits methicillinresistant Staphylococcus aureus biofilm formation and expression of virulence factors. J. Med. Food. 18: 810-817.

25. Mazmanian SK, Ton-That H, Su K, Schneewind O. 2002. An iron-regulated sortase anchors a class of surface protein during Staphylococcus aureus pathogenesis. Proc. Natl. Acad. Sci. USA 99: 2293-2298.

26. Ton-That H, Liu G, Mazmanian SK, Faull KF, Schneewind O. 1999. Purification and characterization of sortase, the transpeptidase that cleaves surface proteins of Staphylococcus aureus at the LPXTG motif. Proc. Natl. Acad. Sci. USA 96: 12424-12329.

27. Lopes LAA, dos Santos Rodrigues JB, Magnani M, de Souza EL, de Siqueira-Júnior JP. 2017. Inhibitory effects of flavonoids on biofilm formation by Staphylococcus aureus that overexpresses efflux protein genes. Microb. Pathog. 107: 193-197.

28. Oh KB, Oh MN, Kim JG, Shin DS, Shin J. 2006. Inhibition of sortase-mediated Staphylococcus aureus adhesion to fibronectin 
via fibronectin-binding protein by sortase inhibitors. Appl. Microbiol. Biotechnol. 70: 102-106.

29. Sinha B, François PP, Nüsse O, Foti M, Hartford OM, Vaudaux P, et al. 1999. Fibronectin-binding protein acts as Staphylococcus aureus invasin via fibronectin bridging to integrin alpha5beta1. Cell. Microbiol. 1: 101-117.

30. O'Neill E, Pozzi C, Houston P, Humphreys H, Robinson DA, Loughman A, et al. 2008. A novel staphylococcus aureus biofilm phenotype mediated by the fibronectin-binding proteins, FnBPA and FnBPB. J. Bacteriol. 190: 3835-3850.

31. Asadollahi P, Farahani NN, Mirzaii M, Khoramrooz SS, Van Belkum A, Asadollahi K, et al. 2018. Distribution of the most prevalent spa types among clinical isolates of methicillin-resistant and -susceptible Staphylococcus aureus around the world: a review. Front Microbiol. 9: 163.

32. Sibbald MJ, Ziebandt AK, Engelmann S, Hecker M, De JA, Harmsen HJ, et al. 2006. Mapping the pathways to staphylococcal pathogenesis by comparative secretomics. Microbiol. Mol. Biol. Rev. 70: 755-788.

33. Schneewind O, Mihaylova-Petkov D, Model P. 1993. Cell wall sorting signals in surface proteins of gram-positive bacteria. EMBO J. 12: 4803-4811.

34. Lowy FD. 1998. Staphylococcus aureus infections. N. Engl. J. Med. 339: 520-532.

35. Allen H, Donato J, Wang H, Cloud-Hansen K, Davies J, Handelsman J. 2010. Call of the wild: antibiotic resistance genes in natural environments. Nat. Rev. Microbiol. 8: 251-259.

36. Foster TJ, Geoghegan JA, Ganesh VK, Höök M. 2014. Adhesion, invasion and evasion: the many functions of the surface proteins of Staphylococcus aureus. Nat. Rev. Microbiol. 12: $49-62$.

37. Geoghegan JA, Foster TJ. 2015. Cell wall-anchored surface proteins of Staphylococcus aureus: many proteins, multiple functions. Curr. Top. Microbiol. Immunol. 409: 95-120.

38. Wang L, Bi C, Cai H, Liu B, Zhong X, Deng X, et al. 2015.
The therapeutic effect of chlorogenic acid against Staphylococcus aureus infection through sortase A inhibition. Front Microbiol. 6: 1031.

39. Kang SS, Kim JG, Lee TH, Oh KB. 2006. Flavonols inhibit sortases and sortase-mediated Staphylococcus aureus clumping to fibrinogen. Biol. Pharm. Bull. 29: 1751-1755.

40. Yang WY, Kim CK, Ahn CH, Kim H, Shin J, Oh KB. 2016. Flavonoid glycosides inhibit sortase A and sortase Amediated aggregation of streptococcus mutans, an oral bacterium responsible for human dental caries. J. Microbiol. Biotechnol. 26: 1566-1569.

41. Liu B, Chen F, Bi C, Wang L, Zhong X, Cai H, et al. 2015. Quercitrin, an inhibitor of sortase A, interferes with the adhesion of Staphylococcal aureus. Molecules 20: 6533-6543.

42. Bi C, Dong X, Zhong X, Cai H, Wang D, Wang L. 2016. Acacetin protects mice from staphylococcus aureus bloodstream infection by inhibiting the activity of sortase A. Molecules 21: 1285 .

43. Cucarella C, Solano C, Valle J, Amorena B, Lasa Í, Penadés JR. 2001. Bap, a Staphylococcus aureus surface protein involved in biofilm formation. J. Bacteriol. 183: 2888-2896.

44. Corrigan RM, Rigby D, Handley P, Foster TJ. 2007. The role of Staphylococcus aureus surface protein SasG in adherence and biofilm formation. Microbiology 153: 2235-2246.

45. Moks T, Abrahmsén L, Nilsson B, Hellman U, Sjöquist J, Uhlén M. 1986. Staphylococcal protein A consists of five IgG-binding domains. Eur. J. Biochem. 156: 637-643.

46. Dedent AC, Mcadow M, Schneewind O. 2007. Distribution of protein A on the surface of Staphylococcus aureus. J. Bacteriol. 189: 4473-4484.

47. Mazmanian SK, Liu G, Jensen ER, Lenoy E, Schneewind O. 2000. Staphylococcus aureus sortase mutants defective in the display of surface proteins and in the pathogenesis of animal infections. Proc. Natl. Acad. Sci. USA 97: 5510-5515. 